\title{
Aberrant Overexpression of Heterogeneous Nuclear Ribonucleoprotein k in Urinary Bladder Cancer Neoplasms
}

Rania Mahmoud ${ }^{1}$, Gehan Hammad ${ }^{1 *}$, Tarek Aboushousha ${ }^{2}$ and Ashraf Bakkar

${ }^{1}$ Faculty of Biotechnology, October Universities for Modern Sciences and Arts, Cairo, Egypt

${ }^{2}$ Department of Pathology, Theodore Bilharz Research Institute, Cairo, Egypt

\begin{abstract}
Bladder cancer remains one of the most prevalent cancers and is considered to be one of the primary causes of morbidity and mortality worldwide. Nonetheless, the treatment and management of this malignancy can be very prosperous only if disease is detected early. Early diagnosis of Bladder cancer is critically important to alleviate morbidity and mortality rates associated with recurrent disease, and to meliorate the chances of a prosperous outcome. Heterogeneous nuclear ribonucleoprotein k (hnRNPK), are multifunctional proteins that are aberrantly overexpressed in various human cancers, and have demonstrated roles in tumorigenesis. Unfortunately, there have been no reports indicating its role in bladder cancer. This study was subsequently aimed at evaluating the expression of hnRNPK in bladder cancer, and determining a correlation of this expression on the basis of various histopathological parameters including type, grade and stage, as well as Bilharzial association. Fifty-eight urinary bladder biopsy specimens were obtained and stained with H\&E and analyzed using immunohistochemistry. The results showed that hnRNPK expression patterns were differentially expressed in benign, dysplastic and malignant lesions. The results also demonstrated that aberrant overexpression in Urinary Bladder Carcinoma tissue is correlated with a poor prognosis and thus elucidated the potential role of hnRNPK as a novel diagnostic and prognostic marker for bladder cancer.
\end{abstract}

Keywords: Urinary bladder neoplasms; hnRNPK; Immunohistochemistry; Pathology

\section{Introduction}

Bladder cancer continues to represent a major health threat, considering the fact that it is one of the major foundations of morbidity and mortality worldwide, accounting for nearly 429,800 new incidence cases and 165,100 deaths per year, it is one of the most common malignant neoplasms in the urological system and is considered as the fourth most prevalent neoplasm in males [1,2]. In Egypt, bladder malignancies are the most common among urinary system malignant tumors $(90.71 \%)$ and the third among all malignancies [3]. Bladder cancer encompasses a wide spectrum of malignancies; yet its main histological type is urothelial carcinoma, which mostly develops along two main, largely independent but rather overlapping biological pathways, commonly known as papillary and non-papillary tumors. Where, papillary tumors are usually instigated by the dispersal of flat hyperplastic urothelial alterations, also termed low-grade intraurothelial neoplasia, and are characterized by superficial non-invasive papillary protrusions [4]. Although it is very unlikely for these tumors to metastasize, they have a significantly high recurrence propensity. Whereas, Non-papillary tumors develop from Maneoplasia. Non-papillary carcinomas are usually characterized by their aggressive invasion through the bladder wall and their ability to metastasize to regional lymph [5].

The other type of carcinoma is squamous cell carcinoma (SCC). SCC is primarily categorized into two subtypes; SCC associated with bilharzia infection (B-SCC) and SCC non-Bilharzia associated squamous cell carcinoma (NB-SCC) [6]. B-SCC and NB-SCC differ in their epidemiology, natural history, and clinicopathological features. B-SCC is predominantly and prevalently found in regions where Bilharzia is endemic [7], such territories include the Middle East, in particular Egypt, and Southeast Asia [8].

Approximately $75 \%$ of newly diagnosed Urinary Bladder Carcinomas are non-muscle invasive papillary tumors [9], while the residual $30 \%$ of newly diagnosed patients have progressed and highly invasive carcinomas [10]. Early Detection and diagnosis of Urinary Bladder Carcinomas cancer is critical to alleviating the rising morbidity and mortality rates associated with this recurrent disease, and to meliorate the chances of a prosperous outcome.

Heterogeneous nuclear ribonucleoprotein $\mathrm{K}$ (hnRNPK), is protein family, and is considered as a vital RNA- and DNA-binding protein. Structurally, it is comprised of three successive K homologue domains that are accountable for the binding of RNA or single-stranded DNA [11]. While biologically, the complexity and diversity of HnRNA renders them multifunctional, where they are not only involved in the development of heterogeneous nuclear RNA into mature RNA [12], but also interact with various molecules essential to regulating gene expression and signal transduction, including chromatin remodeling, DNA transcription, pre-mRNA processing, alternative RNA splicing, and translation, Hence intrinsically regulating their fate [13].

hnRNPK protein is aberrantly overexpressed in various human cancers [14] including colorectal, pancreatic, hepatic, prostate and renal cancer. Heterogeneous nuclear ribonucleoprotein $\mathrm{K}$ allegedly plays a vital role in cancer prognosis, and aberrant levels of its expression correlates with an unfortunate clinical outcome [15]. Several studies have established that hnRNPK were able to endorse metastases through up-regulating matrix metalloproteinas [16,17]. Knockdown of hnRNPK repressed proliferation in pancreatic, colorectal and renal cancer.

*Corresponding author: Gehan Hammad, Faculty of Biotechnology, October Universities for Modern Sciences and Arts, Cairo, Egypt, Tel: +201222375135; E-mail: gihan-hammad@live.com

Received August 25, 2018; Accepted January 21, 2019; Published January 23, 2019

Citation: Mahmoud R, Hammad G, Aboushousha T, Bakkar A (2019) Aberrant Overexpression of Heterogeneous Nuclear Ribonucleoprotein k in Urinary Bladder Cancer Neoplasms. J Cancer Sci Ther 11: 014-019. doi: 10.4172/1948-5956.1000576

Copyright: ( 2019 Mahmoud R, et al. This is an open-access article distributed under the terms of the Creative Commons Attribution License, which permits unrestricted use, distribution, and reproduction in any medium, provided the original author and source are credited. 
Citation: Mahmoud R, Hammad G, Aboushousha T, Bakkar A (2019) Aberrant Overexpression of Heterogeneous Nuclear Ribonucleoprotein k in Urinary Bladder Cancer Neoplasms. J Cancer Sci Ther 11: 014-019. doi: 10.4172/1948-5956.1000576

Given its ubiquitous functions and unique aberrant overexpression in the vast majority of various types of cancer, this study aimed to evaluate the peculiar behavior of hnRNPK in correlation with bladder cancer and associate its immunohistochemical expression to various histopathological parameters including type, grading and staging as well as bilharzial association of the cancer. In addition to determining its clinical significance and characterizing its potential role as a diagnostic marker for bladder cancer.

\section{Materials and Methods}

\section{Patients and clinical pathology data}

The study included fifty-eight $(\mathrm{n}=58)$ urinary bladder biopsy specimens from patients suffering from urinary symptoms at the hospital of Theodor Bilharz Research Institute (TBRI), Cairo, Egypt, seeking medical advice for their urinary symptoms. With Ethical consent, all patients were cystoscopically examined and biopsied for histopathological diagnosis. Tissue sections were obtained from their archival material kept in the pathology department of TBRI All samples were evaluated and histologically diagnosed by expert pathologists using hematoxylin and eosin stained sections, with special reference to type of malignancy, tumor grade and invasiveness (stage) as well as positive or negative association with Bilharzia.

\section{Immunohistochemical (IHC) staining and scoring analyses}

Briefly, paraffin sections of bladder cancer tissues and normal

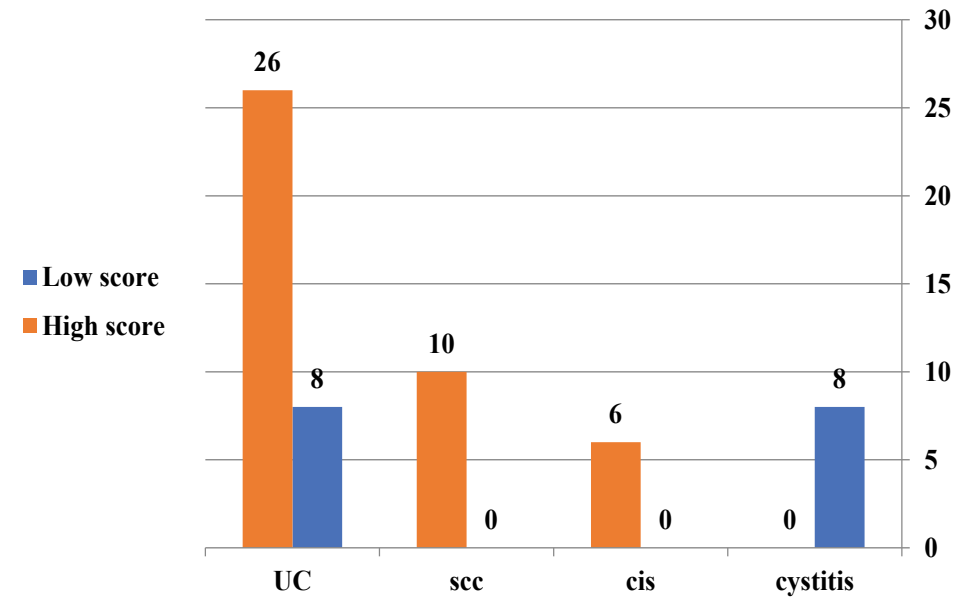

Figure 1: Graphic representation of expression score differences of hnRNPK between all studied groups, based on immunostaining intensities for the percentages of positive cells.
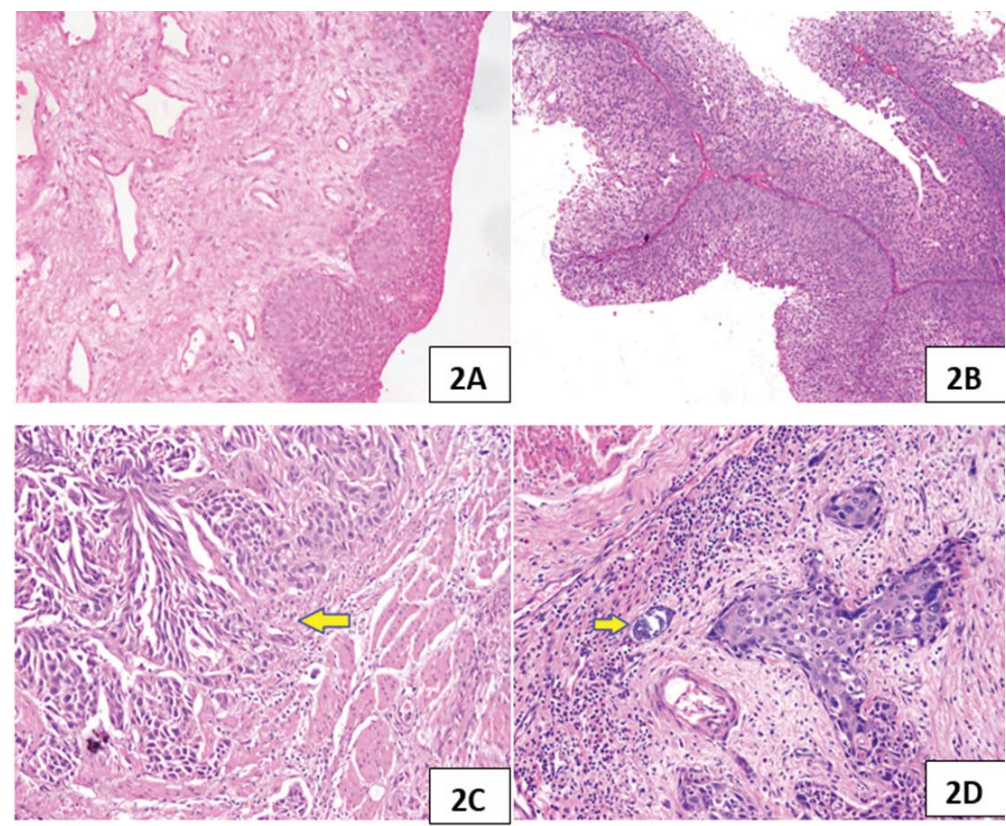

Figure 2: (A) Section in case of chronic cystitis showed mild urothelial hyperplasia as well as mild mononuclear cellular infiltration of the lamina associated with prominent vascular pattern. (H\&E stain, 200x), (B) Section in low grade papillary urothelial carcinoma. (H\&E stain, 100x), (C) Section in case of high-grade urothelia carcinoma invading the muscle propria (T2) (yellow arrow). (H\&E stain, 200x), (D) Section in case of invasive squamous cell carcinoma showing calcified bilharzia ova (yellow arrow). (H\&E stain, 200x). 
Citation: Mahmoud R, Hammad G, Aboushousha T, Bakkar A (2019) Aberrant Overexpression of Heterogeneous Nuclear Ribonucleoprotein k in Urinary Bladder Cancer Neoplasms. J Cancer Sci Ther 11: 014-019. doi: 10.4172/1948-5956.1000576

tissues were first deparaffinized and hydrated. Microwave antigen retrieval was performed for all antibodies, and endogenous peroxidase activity was blocked by incubating the slides in $0.3 \% \mathrm{H}_{2} \mathrm{O}_{2}$. After serial incubation with primary antibodies and secondary antibody, sections were developed with peroxidase and 3,30-diaminobenzidine tetrahydrochloride. The sections were then counterstained with hematoxylin and mounted in non-aqueous mounting medium. Anti-hnRNPK antibody (1:50; sc-28380; Santa Cruz Biotechnology, Santa Cruz, CA, USA) was used to detect hnRNPK expression in the specimens. Human prostate cancer tissues were used as positive controls to test hnRNPK antibody for IHC staining. Negative controls were created by replacing the primary antibody with non-immune immunoglobulin G (IgG; DAKO, Glostrup, Copenhagen, Denmark). Heterogeneous nuclear ribonucleoprotein K expression in the bladder cancer specimens was blind-quantified by two pathologists using the following scoring system [18]. The immunostaining intensity of each sample was graded as negative $=0$, weak $=1$, moderate $=2$, or strong $=3$. The proportion of positively staining cells was assessed as the percentage. The score was then calculated as the intensity score multiplied by the percentage of cells stained (score = intensity $9 \%$ of positive cells). The samples were classed as low (score $<140$ ) or high (score $\geq 140$ ) hnRNPK expression. All sections were assessed and scored. The sections were examined by using light microscope [Scope A1, Axio, Zeiss, Germay]. Photomicrographs were taken using a microscope-camera [AxioCam, MRc5, Zeiss, Germany].

\section{Statistical analyses}

Statistical analyses were performed using the SPSS software (SPSS Standard version 20.0; SPSS Inc., Chicago, IL, USA). Results were characterized as mean \pm SD. Two-tailed Student's t-tests and one-way ANOVA were used to evaluate the results. Comparison of difference in percentages between groups was evaluated using Pearson's Chi square test. Spearman rho test was used for investigating the correlations between the different parameters. Differences were considered statistically significant at $\mathrm{p}<0.05$ and $\mathrm{p}<0.01$.

\section{Results}

The present study investigated the potential correlation of hnRNPK with bladder cancer to various histopathological parameters including type, grading and staging, and bilharzial association. The total sample specimens $(n=58)$ were grouped by and included; eight cases of chronic cystitis $(\mathrm{n}=8)$, six cases of severe dysplasia and carcinoma in situ $(\mathrm{n}=6)$, ten cases of squamous cell carcinoma $(n=8)$ and thirty-four cases of urothelial carcinoma $(n=34)$. For all investigated groups, the mean percentages, intensities, and scores accounting for hnRNPK expression as well as average incidence based on age was analyzed using ANOVA (Table 1). Age incidences demonstrated statistical significance at $(\mathrm{p}<0.001)$. while immunohistochemistry showed significant differences at $(p<0.05)$ between all examined groups for the percentages and scores of cellular heterogeneous nuclear RNA expression, and non-significant with regards to hnRNPK intensity $(\mathrm{p}>0.1)$.

Immunostaining intensities were classified into low or high scores on the basis of positively stained cells. The results demonstrated the lowest intensities of hnRNPK expression in cases of cystitis when compared to other investigated groups of bladder cancer, the positive staining was significantly different $(\mathrm{p}<0.05)$. In squamous cell carcinoma a high expression score is noted, and with regards to urothelial carcinoma (76.5\%) showed high scores of hnRNPK expression (Figure 1).

Analysis for non-papillary urothelial carcinoma showed a significantly higher value of hnRNPK expression in parameters that included percentage, intensity, and scores when statistically compared to the papillary variant. And the associated bladder cancer groups, where analyzed for a pattern of hnRNPK expression with bilharzia, showed a higher hnRNPK percentage $(\mathrm{p}<0.05)$ and hnRNPK score
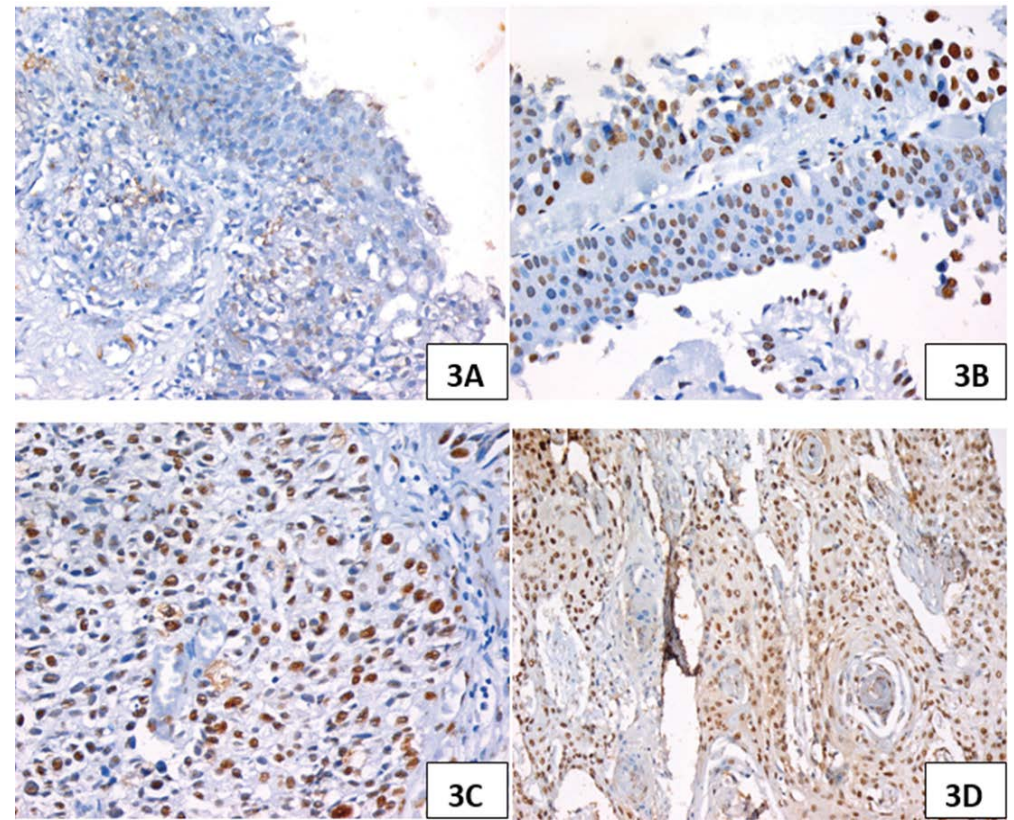

Figure 3: (A) Section in case of chronic cystitis with mild urothelial hyperplasia showing mild cellular expression of HnRNA. (IHC for HnRNA, 200x), (B) Section in case of low grade papillary urothelial carcinoma, showing moderate cellular expression of HnRNA. (IHC for HnRNA, 200x), (C) Section in high grade non-papillary urothelial carcinoma showing high score of cellular expression of HnRNA. (IHC for HnRNA, 400x), (D) Section in squamous cell carcinoma showing marked cellular expression of HnRNA in malignant cells. (IHC for HnRNA, 200x). 
Citation: Mahmoud R, Hammad G, Aboushousha T, Bakkar A (2019) Aberrant Overexpression of Heterogeneous Nuclear Ribonucleoprotein k in Urinary Bladder Cancer Neoplasms. J Cancer Sci Ther 11: 014-019. doi: 10.4172/1948-5956.1000576

( $>0.6)$, as well as a lower hnRNPK expression intensity ( $\mathrm{p}>0.5)$ when compared to non-bilharzia associated bladder cancer cases (Table 2).

Upon the analysis of tumor grade a directly proportional interpretation can be deduced between hnRNPK expression and grading, where high grades of bladder cancer showed significantly higher parameters of expression when compared to low grade tumors. And in interpreting staging effects, bladder cancer cases with muscle invasion showed significantly higher parameters of hnRNPK expression compared to non-muscle invasive tumors (Table 3 ).

Tumor grade shows a highly significant correlation between hnRNPK expression percentage and score $(r=0.545$ and 0.474 respectively, $\mathrm{p}<0.01)$, while it shows significant correlation with hnRNPK intensity $(\mathrm{r}=$ $0.344, \mathrm{p}<0.05)$. Tumor stage shows high significant correlation with all parameters of hnRNPK expression ( $\mathrm{p}<0.01)$ (Figures 2 and 3 ).

\begin{tabular}{|c|c|c|c|c|c|}
\hline \multicolumn{2}{|c|}{ Diagnosis } & hnRNPK Percent & hnRNPK Intensity & hnRNPK Score & $\begin{array}{c}\text { Patient } \\
\text { Age }\end{array}$ \\
\hline \multirow{3}{*}{ Cystitis } & Mean & 30.84 & 2.11 & 60.24 & 25.10 \\
\hline & $\mathrm{N}$ & 8 & 8 & 8 & 8 \\
\hline & Std. Deviation & 0.53 & 0.17 & .07 & 0.44 \\
\hline \multirow{3}{*}{ CIS } & Mean & 90.71 & 3.64 & 270.67 & 66.12 \\
\hline & $\mathrm{N}$ & 6 & 6 & 6 & 6 \\
\hline & Std. Deviation & 0.12 & .57 & 0.19 & 0.25 \\
\hline \multirow{3}{*}{ scc } & Mean & 78.0000 & 2.2000 & 171.28 & 65.4000 \\
\hline & $\mathbf{N}$ & 10 & 10 & 10 & 10 \\
\hline & Std. Deviation & 5.37484 & .42164 & 30.25815 & 10.42646 \\
\hline \multirow{3}{*}{ UC } & Mean & 66.7647 & 2.2941 & 163.2353 & 59.5882 \\
\hline & $\mathbf{N}$ & 34 & 34 & 34 & 34 \\
\hline & Std. Deviation & 27.13274 & .67552 & 78.04313 & 9.89679 \\
\hline \multirow{3}{*}{ Total } & Mean & 68.5417 & 2.2917 & 165.85 & 59.6250 \\
\hline & $\mathbf{N}$ & 58 & 58 & 58 & 58 \\
\hline & Std. Deviation & 25.03101 & .61742 & 73.48469 & 12.22419 \\
\hline$p$-value & & $<0.05$ & $>0.1$ & $<0.05$ & $<0.001$ \\
\hline
\end{tabular}

Table 1: Differences in mean percentages, intensities and scores of hnRNPK expression as well as in age incidence in examined groups.

\begin{tabular}{|c|c|c|c|c|c|}
\hline & Papillary pattern & $\mathbf{N}$ & Mean & Std. Deviation & $p$-value \\
\hline \multirow{2}{*}{ hnRNPK Percent } & Non-papillary UC & 22 & 80.9091 & 14.27922 & \multirow{2}{*}{$p<0.001$} \\
\hline & Papillary UC & 14 & 47.8571 & 29.98168 & \\
\hline \multirow{2}{*}{ hnRNPK Intensity } & Non-papillary & 22 & 2.5455 & 0.50965 & \multirow{2}{*}{$\mathrm{p}<0.05$} \\
\hline & Papillary UC & 14 & 2.0000 & 0.78446 & \\
\hline \multirow{2}{*}{ hnRNPK Score } & Non-papillary & 22 & 203.1818 & 44.84220 & \multirow{2}{*}{$p<0.01$} \\
\hline & Papillary UC & 14 & 115.7143 & 93.98971 & \\
\hline & \multicolumn{5}{|c|}{ Bilharzia association } \\
\hline \multirow{2}{*}{ hnRNPK Percent } & Negative & 38 & 66.0526 & 27.53764 & \multirow{2}{*}{$p<0.05$} \\
\hline & Positive & 10 & 78.0000 & 5.37484 & \\
\hline \multirow{2}{*}{ hnRNPK Intensity } & Negative & 38 & 2.3158 & .66191 & \multirow{2}{*}{$P>0.5$} \\
\hline & Positive & 10 & 2.2000 & .42164 & \\
\hline \multirow{2}{*}{ hnRNPK Score } & Negative & 38 & 163.4211 & 81.39083 & \multirow{2}{*}{$P>0.6$} \\
\hline & Positive & 10 & 171.0000 & 30.25815 & \\
\hline
\end{tabular}

Table 2: Difference in hnRNPK expression parameters between non-papillary and papillary urothelial carcinoma (UC).

\begin{tabular}{|c|c|c|c|c|}
\hline \multicolumn{2}{|c|}{ Grade of tumor (Low/High) } & hnRNPK Percent & hnRNPK Intensity & hnRNPK Score \\
\hline \multirow{3}{*}{ High } & Mean & 79.1667 & 2.4444 & 191.6667 \\
\hline & $\mathrm{N}$ & 36 & 36 & 36 \\
\hline & Std. Deviation & 11.37039 & .50395 & 41.09223 \\
\hline \multirow{3}{*}{ Low } & Mean & 25.45 & 1.5000 & 45.556 \\
\hline & $\mathrm{N}$ & 8 & 8 & 8 \\
\hline & Std. Deviation & 16.03567 & .53452 & 37.41657 \\
\hline p-value (t-test) & & $p<0.0001$ & $p<0.0001$ & $p<0.0001$ \\
\hline \multicolumn{5}{|c|}{ Muscle Invasion } \\
\hline \multirow{3}{*}{ Non-invasive } & Mean & 43.3333 & 1.8333 & 98.3333 \\
\hline & $\mathrm{N}$ & 12 & 12 & 12 \\
\hline & Std. Deviation & 30.55050 & .71774 & 92.91573 \\
\hline \multirow{3}{*}{ Invasive } & Mean & 79.0625 & 2.4375 & 190.595 \\
\hline & $\mathrm{N}$ & 32 & 32 & 32 \\
\hline & Std. Deviation & 11.53099 & .50402 & 36.54427 \\
\hline p-value (t-test) & & $p<0.0001$ & $P=0.0031$ & $p<0.0001$ \\
\hline
\end{tabular}

Table 3: Difference in mean percentage, intensity and score of hnRNPK expression in relation to tumor grade and stage (muscle invasion). 


\section{Discussion}

Aberrant overexpression of hnRNPK has been previously reported in various cancers including those of the colon [17], lung [19], renal [20] and pancreas [13]. Several studies have concluded that hnRNPK is robustly involved in the development, and the progression of cancer through various biological events including transcriptional regulation, translational control chromatin remodeling, and cellular signal transduction [14]. However, there has been no previous report regarding the behavior and expression of hnRNPK underlying tumorigenesis and advancement in bladder cancer.

The performed immunohistochemistry study showed significant difference between groups considering the percentage and score of cellular expression of heterogeneous nuclear RNA and non-significant difference concerning hnRNPK intensity. As shown in Figure 1, both of the urothelial cell carcinoma and squamous cell carcinoma cases showed relatively high scores of hnRNPK compared to cystitis and CIS. Conversely, there were significant differences regarding all cases of cystitis, which showed a relatively low score of hnRNPK expression compared to other cases of bladder cancer. The results documented in Table 1 illustrates that predominant expression of hnRNPK in malignant cases for instance; urothelial and squamous cell carcinoma, are significantly higher than those of cystitis cases. These findings are in agreement with Ayham et al. [21] where there was a significant decrease in nuclear hnRNPK expression levels in primary colon tumor compared to primary tumor to lymph node colon metastasis and in alignment with Guo et al. [22] as they were able to distinguish between early and late hepatocellular carcinoma through the expression and intensity of hnRNPK. In addition to, the conclusion that the intensive hnRNPK activity was restricted to the nucleus compared to the cytoplasm of tumor cells, whereas faint nuclear immunostaining was observed in cirrhotic tissues. The difference in hnRNPK expression parameters between non-papillary and papillary urothelial carcinoma (UC) was also investigated in the present study. As shown in Table 2, non-papillary urothelial carcinoma cases exhibited significantly higher values of all parameters of hnRNPK expression, when compared to the papillary variant. These findings may suggest that the relative score of hnRNPK expression is proportional to the degree of invasiveness and aggressiveness of the tumor. These results can be associated with those of Ayham et al. [21] where the nuclear hnRNPK staining escaladed with the grading and invasiveness of colorectal cancer. These findings could be due to the ability of hnRNPK promote tumor metastasis through the regulation of various extracellular matrix, cell motility, and angiogenesis pathways that resulted in enhanced malignancies and the overexpression of hnRNPK [23].

Table 2 also shows that Bladder cancer cases associated with Bilharzia also showed a higher percentage of hnRNPK expression and hnRNPK score as well as lower hnRNPK expression intensity compared to non-Bilharzia associated bladder cancer. However, there were no significant differences between the expression scores of Bilharzia and non-Bilharzia associated cancers.

Nonetheless, the correlation between hnRNPK expression and pathological grade in human various bladder cancer specimens was also examined. The expression level of hnRNPK in bladder cancer tissues was positively correlated with higher grade tumors, a potent predictor of tumor progression, as shown in Table 3. Moreover, hnRNPK expression was significantly upregulated in bladder cancer cases with distant muscle invasiveness, compared with those without muscle invasion. These previous results are in line with the findings of Otoshi et al. [20] who demonstrated a significant positive correlation between hnRNPK staining scores and tumor aggressiveness in renal cell carcinoma. Also, in this immunohistochemistry analysis, the rate of positive hnRNPK staining in the cytoplasmic constituent was significantly higher in muscle invasive tissues compared to nonmuscle invasive tissues. These findings are also in agreement with a study conducted by Carpenter et al. [17] on colorectal cancer where the significant expression of hnRNPK protein has been shown to be positively associated with both, cancer progression and patient prognosis, where the significant high expressions of hnRNPK is frequently correlated with poor prognosis [24-26].

\section{Conclusion}

In conclusion, although hnRNPK is considered a promoter of various kinds of malignancies, its underlying actions in bladder cancer remains unclear. In this study, the function and the underlying mechanism of hnRNPK expressions in bladder carcinogenesis was investigated. The immunohistochemistry findings of this study elucidated a possible correlation between hnRNPK expression and the progression of various urinary bladder lesions into bladder carcinoma. hnRNPK expression patterns may hold a significant diagnostic value to evaluate possible progression of urinary bladder lesions to the dysplastic and/or malignant lesions. The results also demonstrated that aberrant enhancement of hnRNPK expression in bladder cancer tissues was associated with poor prognosis of bladder cancer patients and thus can be a potential diagnostic and prognostic marker for bladder cancer.

\section{References}

1. Torre LA, Bray F, Siegel RL, Ferlay J, Lortet-Tieulent J, et al. (2015) Global cancer statistics, 2012. CA Cancer J Clin 65: 87-108.

2. Siegel RL, Miller KD, Jemal A (2017) Cancer statistics, 2017. CA Cancer J Clin 67: 7-30.

3. Harb OA, Haggag R, Ali MM, El Shorbagy S, Abdelbary AM, et al. (2017) The prognostic role of NEDD9 and P38 protein expression levels in urinary bladder transitional cell carcinoma. J Oncol 6: 205

4. Anastasiadis A, De Reijke TM (2012) Best practice in the treatment of nonmuscle invasive bladder cancer. Ther Adv Urol 4: 13-32.

5. McConkey DJ, Lee S, Choi W, Tran M, Majewski T, et al. (2010) Molecular genetics of bladder cancer: Emerging mechanisms of tumor initiation and progression. Urol Oncol 28: 429-440.

6. Martin JW, Carballido EM, Ahmed A, Farhan B, Dutta R, et al. (2016 Squamous cell carcinoma of the urinary bladder: Systematic review of clinical characteristics and therapeutic approaches. Arab J Urol 14: 183-191.

7. Rausch S, Hofmann R, Von Knobloch R (2012) Non-bilharzial squamous cell carcinoma and transitional cell carcinoma with squamous differentiation of the lower and upper urinary tract. Urol Ann 4: 14-18.

8. Olveda DU, Li Y, Olveda RM, Lam AK, Chau TN, et al. (2013) Bilharzia: Pathology, diagnosis, management and control. Trop Med Surg 1: 135.

9. Isharwal S, Konety B (2015) Non-muscle invasive bladder cancer risk stratification. Indian J Urol 31: 289-296.

10. Veeratterapillay R, Heer R, Johnson MI, Persad R, Bach C (2016) High-risk non-muscle-invasive bladder cancer-therapy options during intravesical BCG shortage. Curr Urol Rep 17: 68

11. Chaudhury A, Chander P, Howe PH (2010) Heterogeneous nuclea ribonucleoproteins (hnRNPs) in cellular processes: Focus on hnRNP E1's multifunctional regulatory roles. RNA 16: 1449-1462.

12. Dinh PX, Das A, Franco R, Pattnaik AK (2013) Heterogeneous nuclear ribonucleoprotein $\mathrm{k}$ supports vesicular stomatitis virus replication by regulating cell survival and cellular gene expression. J Virol 87: 10059-10069.

13. Zhou R, Shanas R, Nelson MA, Bhattacharyya A, Shi J (2010) Increased expression of the heterogeneous nuclear ribonucleoprotein $\mathrm{k}$ in pancreatic cancer and its association with the mutant p53. Int J Cancer 126: 395-404. 
Citation: Mahmoud R, Hammad G, Aboushousha T, Bakkar A (2019) Aberrant Overexpression of Heterogeneous Nuclear Ribonucleoprotein k in Urinary Bladder Cancer Neoplasms. J Cancer Sci Ther 11: 014-019. doi: 10.4172/1948-5956.1000576

14. Lu J, Gao FH (2016) Role and molecular mechanism of heterogeneous nuclear ribonucleoprotein $\mathrm{K}$ in tumor development and progression. Biomed Rep 4: 657-663

15. Yang R, Zeng $Y, X u H$, Chen Z, Xiang M, et al. (2016) Heterogeneous nuclear ribonucleoprotein $\mathrm{K}$ is overexpressed and associated with poor prognosis in gastric cancer. Oncol Rep 36: 929-935.

16. Chung IC, Chen LC, Chung AK, Chao M, Huang HY, et al. (2014) Matrix metalloproteinase 12 is induced by heterogeneous nuclear ribonucleoprotein $\mathrm{K}$ and promotes migration and invasion in nasopharyngeal carcinoma. BMC Cancer 14: 348 .

17. Carpenter B, McKay M, Dundas SR, Lawrie LC, Telfer C, et al. (2006) Heterogeneous nuclear ribonucleoprotein $\mathrm{K}$ is over expressed, aberrantly localised and is associated with poor prognosis in colorectal cancer. $\mathrm{Br} \mathrm{J}$ Cancer 95: 921-927.

18. Chen X, Gu P, Xie R, Han J, Liu H, et al. (2016) Heterogeneous nuclear ribonucleoprotein $\mathrm{K}$ is associated with poor prognosis and regulates proliferation and apoptosis in bladder cancer. J Cell Mol Med 20: 1-14.

19. Tang F, Li W, Chen Y, Wang D, Han J, et al. (2014) Downregulation of hnRNP $\mathrm{K}$ by RNAi inhibits growth of human lung carcinoma cells. Oncol Lett 7: 10731077.

20. Otoshi T, Tanaka T, Morimoto K, Nakatani T (2015) Cytoplasmic accumulation of heterogeneous nuclear ribonucleoprotein $\mathrm{k}$ strongly promotes tumor invasion in renal cell carcinoma cells. PLoS one 10: e0145769.

21. Ayham A, Brian C, Colin T, Murray GI (2009) Colorectal cancer: immunohistochemical diagnosis with heterogeneous nuclear ribonucleoprotein k. Colorectal Can 25-42.

22. Guo Y, Zhao J, Bi J, Wu Q, Wang X, et al. (2012) Heterogeneous nuclear ribonucleoprotein $\mathrm{K}(\mathrm{hnRNP} \mathrm{K})$ is a tissue biomarker for detection of early Hepatocellular carcinoma in patients with cirrhosis. J Hematol Oncol 5: 37.

23. Gao R, Yu Y, Inoue A, Widodo N, Kaul SC, et al. (2013) Heterogeneous nuclear ribonucleoprotein $k$ (hnRNP-K) promotes tumor metastasis by induction of genes involved in extracellular matrix, cell movement, and angiogenesis. J Biol Chem 288: 15046-15056.

24. Braicu C, Cojocneanu-Petric R, Chira S, Truta A, Floares A, et al. (2015) Clinical and pathological implications of miRNA in bladder cancer. Int $\mathrm{J}$ Nanomedicine 10: $791-800$.

25. Ibrahim AS, Khaled HM, Mikhail NN, Baraka H, Kamel H (2014) Cancer incidence in Egypt: Results of the National population-based cancer registry program. J Cancer Epidemiol pp: 1-18.

26. Inman BA, Stauffer PR, Craciunescu OA, Maccarini PF, Dewhirst MW, et al. (2014) A pilot clinical trial of intravesical mitomycin-C and external deep pelvic hyperthermia for non-muscle-invasive bladder cancer. Int J Hyperthermia 30: 171-175. 